\title{
La cirugía de cadera en el Centro Médico ABC
}

\section{The hip surgery at the ABC Medical Center}

\author{
Javier Camacho Galindo*
}

Citar como: Camacho GJ. La cirugía de cadera en el Centro Médico ABC. An Med ABC. 2021; 66 (2): 80. https://dx.doi.org/10.35366/100473

Una de las subespecialidades de la ortopedia, que ha tenido grandes avances en conocimientos y técnicas en las últimas dos décadas, es la cirugía de cadera; por ser una de las articulaciones más grandes de carga es una de las más estudiadas. Varias patologías y conceptos nuevos cambiaron la forma de estudiar esta articulación, sin duda el pinzamiento femoroacetabular fue uno de los más destacados, descrito por Ganz, quien inició con algunos conceptos, logrando más tarde un cambio radical, desde la exploración física pasando por la biomecánica, hasta las diferentes técnicas quirúrgicas descritas.

No se quedó atrás el tratamiento de la artrosis, en los 80 se pensaba que estaba resuelto el problema con la prótesis de baja fricción descrita por Charnley, durante las décadas siguientes el desarrollo de las técnicas no cementadas ha demostrado que todavía se podía mejorar el sistema de fijación, hasta hacerlo casi predecible en la actualidad. Al mismo tiempo, el avance tecnológico de los biomateriales de fricción de la articulación ayudó a aumentar el tiempo de supervivencia, ya sea polietileno de enlaces cruzados, metal o cerámica.

En este número, dedicado en especial a la cirugía de cadera, podemos leer la gran controversia que existe entre los diferentes abordajes quirúrgicos para artroplastia total, ya que aún no se encuentra el abordaje «ideal»; nos comparten la experiencia junto con las actualidades del ahora famoso y renovado

\footnotetext{
* Cirujano Ortopedista del Centro Médico ABC. Profesor titular de Curso de postgrado de Ortopedia y traumatológica del Centro Médico ABC.
}

Correspondencia: Dr. Javier Camacho Galindo

E-mail: jurcamacho@gmail.com abordaje anterior, así como el estado actual del abordaje antero-lateral. No podía quedarse fuera la parte traumatológica, donde la fractura de cadera es una de las principales patologías tratadas en el Centro Médico ABC, determinar la mortalidad de los tratamientos más frecuentes de la fractura es importante para una adecuada toma de decisión. También se muestran algunos casos de cadera neoplásicos, traumáticos y crónicos que vale la pena revisar y conocer para tener en consideración el tipo de cirugía que se está realizando en nuestro hospital.

Una de las fortalezas del Centro Médico ABC que se menciona en un editorial de 2012 de este mismo medio de difusión es la estimulación de la investigación de las generaciones jóvenes, ahora en este número se presentan dos artículos, uno en el que se investiga la definición del centro de rotación en la población mexicana, el cual es el primero de su género; y el segundo nos muestra los cambios que se tuvieron que realizar en el aprendizaje de la residencia de ortopedia debido a la pandemia COVID-19, así como su impacto académico. El Departamento de Ortopedia y Traumatología busca ser líder en la educación de la próxima generación de cirujanos ortopedistas a través de su residencia médica y programas de formación, que incluyen: exposición completa y a fondo en todas las áreas de la ortopedia, así como experiencia quirúrgica avanzada.

Contribuir al desarrollo del conocimiento de la ortopedia en México mediante la elaboración y publicación de investigaciones no sólo debe ser orgullo de todos nosotros, integrantes del Centro Médico ABC, sino también debe ser el motor para la continua producción de publicaciones de calidad. No olvidemos que somos el ejemplo de muchos otros. 